OPEN ACCESS

Edited by:

Wen Zhang,

Chiba University, Japan

Reviewed by:

Xiao Luo,

China Medical University, China

Liyan Guo,

Jining Medical University, China

*Correspondence:

Kexin Chen

chenkexin@tmu.edu.cn

Specialty section:

This article was submitted to

Nutritional Epidemiology,

a section of the journal

Frontiers in Nutrition

Received: 09 August 2021 Accepted: 06 September 2021

Published: 12 October 2021

Citation:

Liu L, Sheng C, Lyu Z, Dai H and Chen K (2021) Association Between Genetically Proxied Lipid-Lowering

Drug Targets and Renal Cell Carcinoma: A Mendelian Randomization Study.

Front. Nutr. 8:755834. doi: 10.3389/fnut.2021.755834

\section{Association Between Genetically Proxied Lipid-Lowering Drug Targets and Renal Cell Carcinoma: A Mendelian Randomization Study}

\author{
Luyang Liu, Chao Sheng, Zhangyan Lyu, Hongji Dai and Kexin Chen* \\ Department of Epidemiology and Biostatistics, National Clinical Research Center for Cancer, Key Laboratory of Molecular \\ Cancer Epidemiology of Tianjin, Tianjin Medical University Cancer Institute and Hospital, Tianjin, China
}

Observational studies suggested inconsistent associations between lipid-lowering drugs, such as statins, and renal cell carcinoma (RCC) risk. In a two-sample Mendelian randomization (MR) framework, we assessed the causal influence of lipid-lowering agents and circulating lipid traits on overall and sex-specific RCC risk. Genetic variants of six drug-target genes were selected to proxy the effects of low-density lipoprotein cholesterol (LDL-C) lowering therapies. Instrumental variables for circulating lipid traits were constructed from two large genome-wide association studies. We used endpoints for RCC from summary statistics of two studies [International Agency for Research on Cancer [IARC], $N=13,230$; National Cancer Institute [NCI], $N=4,735]$. The robustness of results was assessed through conventional MR sensitivity analyses. Overall, there was no significant association between genetically proxied HMG-CoA reductase (HMGCR) inhibition and $\mathrm{RCC}$ risk [Odds ratio [OR] $=1.42,95 \% \mathrm{Cl}, 0.29-6.99]$. In the sex-stratified analysis, we observed a positive association for genetically proxied drug targets with RCC risk. Specifically, genetically proxied proprotein convertase subtilisin/kexin type 9 (PCSK9) inhibition was associated with a higher risk of $\mathrm{RCC}$ in men $[\mathrm{OR}=2.20[95 \% \mathrm{Cl}$, 1.24-3.89]], and the difference by sex was moderate. This study suggested genetically proxied inhibition of HMGCR was not associated with RCC risk, while genetically proxied PCSK9 inhibition might be associated with a higher risk of RCC in male.

Keywords: lipid-lowering drug, renal cell carcinoma, mendelian randomization, sex-specific, lipoprotein

\section{INTRODUCTION}

According to the estimates of cancer incidence and mortality reported by the International Agency for Research on Cancer (IARC), there were $\sim 430,000$ new cases diagnosed with renal cell carcinoma (RCC) and 180,000 deaths worldwide in 2020 (1). RCC patients are insensitive to conventional cytotoxic chemotherapy, cytokine therapy, and radiotherapy, and the underlying mechanisms are still unclear; moreover, currently, there is no efficient screening strategy for RCC (2). Therefore, primary prevention of RCC is necessary for reducing the disease burden.

Clear cell RCC (ccRCC) is characterized by the accumulation of lipid droplets in the cytoplasm. Both fatty acid synthesis and lipid storage could promote the growth of ccRCC (3). Observational studies also suggested that dyslipidemia might be involved in the carcinogenesis of RCC (4). However, a recent Mendelian randomization (MR) study that incorporated the largest published 
circulating lipid traits genome wide association study (GWAS) and RCC GWAS in European ancestry reported that there was no causal association for the influence of low-density lipoprotein cholesterol (LDL-C), high-density lipoprotein cholesterol (HDLC), total cholesterol (TC), and triglyceride (TG) on RCC risk (5).

Statins are the inhibitors of 3-hydroxy-3-methylglutaryl coenzyme A (HMG-CoA) reductase and are the most commonly prescribed lipid-lowering agents that are widely used in both primary and secondary prevention of cardiovascular diseases (CAD). Recent researches have reported its cancer preventive effects, including promoting apoptosis, suppressing angiogenesis, and inhibiting tumor growth and metastasis (6). However, no increase of HMG-CoA reductase (HMGCR) activity was observed in RCC, making the role of statins in the prevention of RCC confused (7). In addition, growing epidemiological studies have investigated the association between statin use and the risk of RCC (8-11). However, the conclusions of these studies are controversial. Several studies reported a risk reduction of RCC in statin users $(8,9)$; while a nationwide case-control study indicated no chemopreventive effect of long-term use of statin on RCC (10), and another population-based study in Korea reported that statin elevated the risk of kidney cancer (11). Findings of traditional observational studies may be biased due to confounding factors, reverse causal association, and residual confounding, making the interpretation of these findings challenging. Some clinical trials have also explored the effect of lipid-modifying drugs on cancer risk; for example, a phase 3 clinical trial that was designed to assess the clinical efficacy and safety of anacetrapib [cholesteryl ester transfer protein [CETP] inhibitor] has reported a slightly increased but not significant risk of genitourinary cancer [relative risk [RR]: 1.08 , 95\% CI: $0.93-$ 1.27] (12). However, due to limited follow-up periods and small numbers of RCC cases in clinical trials, it is difficult to make causal inferences for the relationship between lipid-lowering therapies and RCC risk.

Mendelian randomization is a method designed for causal inference using genetic variants to construct instrument variables. Genetic variants are randomly allocated at conception, and they are largely independent of potential confounders and reverse causality, which is common in conventional observational studies (13). Thus, MR may minimize confounding factors and provide more credible causal effect estimates without any potentially harmful interventions. With the rapid development of fundamental theory and growth of applications, drug-target MR analysis gradually becomes an efficient tool that can be applied to infer the influence of agents targeting proteinencoding genes, antagonists, agonists, activators, or inhibitors on disease risk (14). Compared to molecule-specific MR analysis, drug-target $\mathrm{MR}$ analysis generated instruments using genetic variants in DNA sequences located within or near genes, which encode the drug target to predict the effect of the corresponding drug. These variants may alter the expression or function of target genes (15). Yamolinsky et al. employed this method and identified a reverse relationship between genetically proxied HMGCR inhibitor and ovarian cancer risk (16). Using a similar study design, Luo et al. found a risk reduction effect of metformin on cardiovascular and cancer risk (17).
In this study, we performed drug-target and molecule-specific MR analyses to estimate the causal effects of circulating lipid traits and variants in genes encoding lipid-modifying drug targets on the risk of RCC under a two-sample MR framework. We aimed to evaluate whether genetically proxied lipid-lowering drugs and circulating lipid traits could influence the risk of RCC in both men and women.

\section{MATERIALS AND METHODS Study Design}

We designed the main analysis and secondary analysis in priori. In the main analysis, we performed drug-target MR analysis to investigate the association of genetically proxied HMGCR (targets of statins), Niemann-Pick C1-Like 1 (NPC1L1, targets of ezetimibe), proprotein convertase subtilisin/kexin type 9 (PCSK9, target of evolocumab and alirocumab), CETP (target of anacetrapib), low density lipoprotein receptor $(L D L R)$, and $A P O B$ (target of mipomersen) inhibitors with overall and sexspecific RCC risk. $L D L R$ was not a specific drug target of any lipid-modifying agent, however, it was involved in the lipid metabolism, thus, we also assessed the role of the LDLR pathway in relation to the risk of RCC in our analysis. In the secondary analysis, we conducted a molecule-specific MR analysis to explore the causal relationship of circulating lipid traits, including LDL-C, HDL-C, TC, TG, ApoA, and ApoB, with overall and sex-specific RCC risk. Details of the study design were shown in Figure 1.

\section{Data Source}

Data used in our study were publicly available from largescale GWASs. All studies were approved by their respective institutional review board and were in concordance with the Declaration of Helsinki. Informed consent was also obtained from each participant.

To generate instrument variables for lipid-lowering drug targets, we obtained summary statistics from a GWAS of LDLC conducted by the Global Lipid Genetics Consortium (GLGC) (18). Participants of European ancestry from 23 studies ( $N$ $=94,595)$ were genotyped with customized GWAS chips and individuals from 37 studies $(N=93,982)$ were genotyped using Metabochip arrays. In both studies, standard quality control and imputation to the 1,000 Genomes Project reference panel were performed. Circulating lipid levels of individuals treated without taking lipid-lowering drugs recently were measured after $8 \mathrm{~h}$ fasting. The Association test of each SNP was performed using linear regression with the inverse normal transformed trait values as the dependent variable and the allele count for each individual as the independent variable (18). To proxy drug-target effects, we applied a linkage disequilibrium (LD) clumping method. Specifically, we selected variants that were located within the $\pm 100 \mathrm{~kb}$ range of each target gene and associated with LDL-C at a genome-wide significant threshold $\left(P<5 \times 10^{-8}\right)$. We then clumped these SNPs according to LD $r^{2} \leq 0.2$ and a physical distance of $250 \mathrm{~kb}$. For instruments with $<3$ SNPs, we further relaxed the $\mathrm{LD} r^{2}$ threshold to 0.40 to enlarge the variance explained by the instruments. Finally, there were 5 SNPs for 


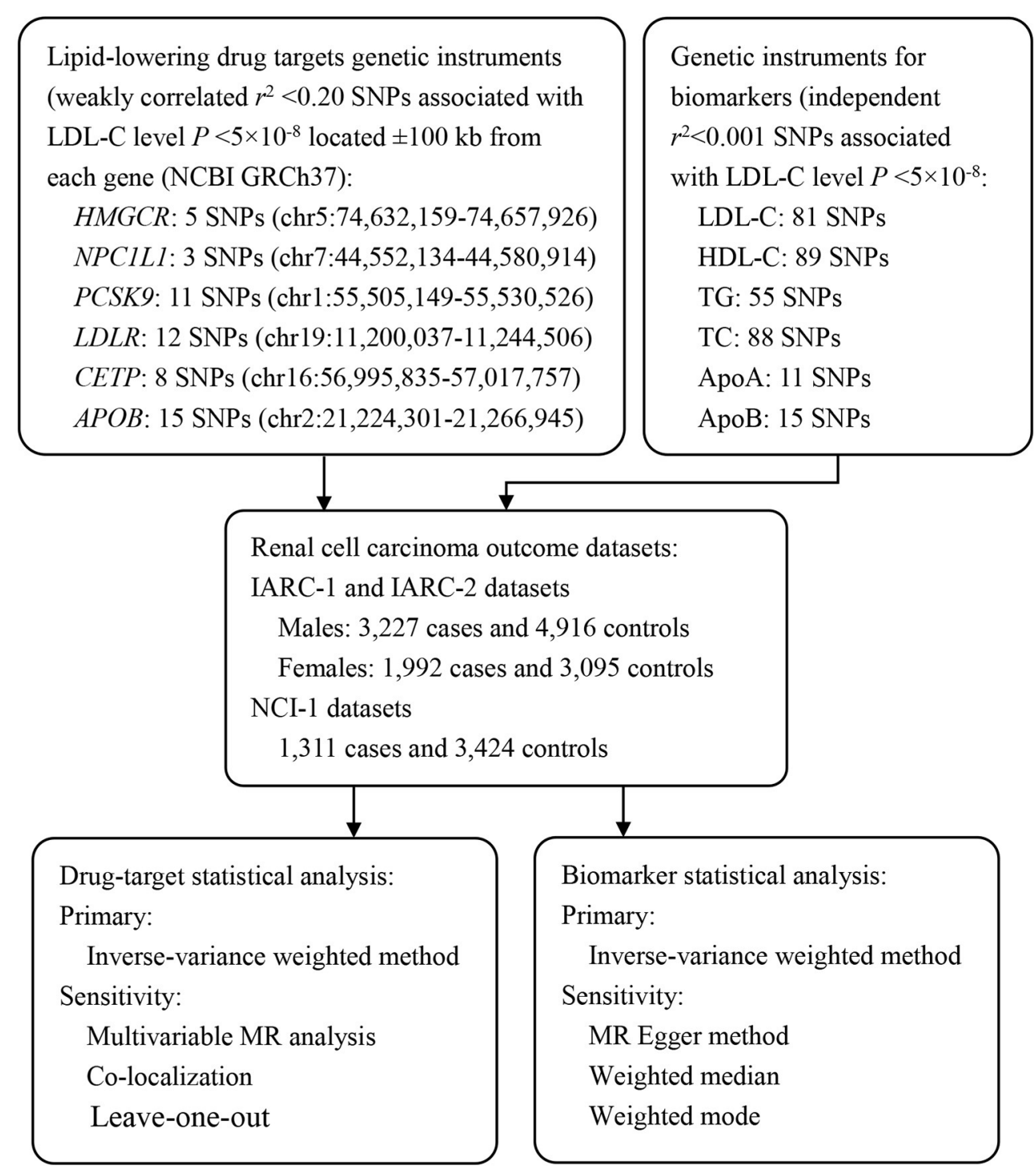

FIGURE 1 | Overview of the study design. To construct instruments for drug targets and circulating biomarkers, summary genetic association data with LDL-C, HDL-C, TG, and TC were obtained from a European ancestry GWAS conducted by GLGC ( $N=188,577)$. These SNPs were then matched with RCC outcome datasets to obtain SNP-outcome associations. After matching SNPs across traits and aligning them into the same effect allele, Mendelian randomization analyses were performed using the IVW method as the primary analyses, and various sensitivity analyses were applied to test Mendelian randomization assumptions (exchangeability and exclusion restriction). HMGCR, 3-Hydroxy-3-Methyl-Glutaryl-Coenzyme A Reductase; NPC1L1, Niemann-Pick C1-Like 1; PCSK9, Proprotein Convertase Subtilisin/Kexin type 9; LDLR, Low Density Lipoprotein Receptor; CETP, Cholesteryl Ester Transfer Protein; APOB, Apolipoprotein B; LDL-C, Low Density Lipoprotein Cholesterol; HDL-C, High Density Lipoprotein Cholesterol; TG, Triglyceride; TC, Total Cholesterol; ApoA, Apolipoprotein A; ApoB, Apolipoprotein B; MR, Mendelian Randomization; IARC, The International Agency for Research on Cancer; NCl, The National Cancer Institute.

proxy of HMGCR, 4 SNPs for NPC1L1, 11 SNPs for PCSK9, 12 SNPs for $L D L R, 8 \mathrm{SNPs}$ for CETP, and 15 SNPs for $A P O B$ (Table 1).

To construct instruments for LDL-C, HDL-C, TG, and TC, we extracted variants associated with each lipid trait at a genomewide significance $\left(P<5 \times 10^{-8}\right)$, LD $r^{2} \leq 0.001$, and a physical distance $\geq 10 \mathrm{Mb}$ from the GLGC GWAS summary data. There were $81,89,55$, and 88 SNPs selected for LDL-C, HDL-C, TG, and TC instruments, respectively (Supplementary Table 2). Moreover, we included another two lipid traits, Apolipoprotein A
(ApoA) and Apolipoprotein B (ApoB), from a recently published large-scale GWAS study that used nuclear magnetic resonance (NMR) metabolomics to quantify circulating metabolic traits with up to 24,924 European individuals (19). We used the same criteria and selected 11 and 15 SNPs for ApoA and ApoB, respectively (Supplementary Table 2 ).

The endpoints for RCC were selected from two RCC GWAS studies. All RCC cases were defined on the basis of the International Classification of Disease for Oncology, Second Edition (ICD-O-2) coded as C64 including all histological 
TABLE 1 | Characteristics of LDL Cholesterol-Lowering Genetic Variants within/near HMGCR, NPC1L1, PCSK9, LDLR, CETP, and APOB gene.

\begin{tabular}{|c|c|c|c|c|}
\hline SNP & EA/NA & EAF & Effect $(95 \% \mathrm{Cl})^{\dagger}$ & $P$-value \\
\hline \multicolumn{5}{|l|}{ HMGCR } \\
\hline rs7711235 & $A / G$ & 0.73 & $-0.038(-0.050,-0.025)$ & $5.00 \times 10^{-10}$ \\
\hline rs3857388 & $\mathrm{T} / \mathrm{C}$ & 0.87 & $-0.042(-0.054,-0.031)$ & $2.20 \times 10^{-11}$ \\
\hline rs10515198 & $\mathrm{G} / \mathrm{A}$ & 0.90 & $-0.060(-0.072,-0.048)$ & $5.99 \times 10^{-22}$ \\
\hline rs12916 & $\mathrm{T} / \mathrm{C}$ & 0.57 & $-0.073(-0.081,-0.066)$ & $7.79 \times 10^{-78}$ \\
\hline rs12173076 & $\mathrm{T} / \mathrm{G}$ & 0.88 & $-0.065(-0.076,-0.054)$ & $2.33 \times 10^{-27}$ \\
\hline \multicolumn{5}{|l|}{ NPC1L1 } \\
\hline rs217386 & $A / G$ & 0.81 & $-0.049(-0.058,-0.039)$ & $1.92 \times 10^{-21}$ \\
\hline rs2073547 & $A / G$ & 0.41 & $-0.036(-0.044,-0.029)$ & $1.20 \times 10^{-19}$ \\
\hline rs17655652 & $\mathrm{C} / \mathrm{T}$ & 0.29 & $-0.028(-0.037,-0.019)$ & $2.18 \times 10^{-10}$ \\
\hline rs7791240 & $\mathrm{T} / \mathrm{C}$ & 0.91 & $-0.043(-0.055,-0.030)$ & $1.84 \times 10^{-10}$ \\
\hline \multicolumn{5}{|l|}{ PCSK9 } \\
\hline rs2479394 & $A / G$ & 0.71 & $-0.039(-0.047,-0.031)$ & $1.58 \times 10^{-19}$ \\
\hline rs11206510 & $\mathrm{C} / \mathrm{T}$ & 0.15 & $-0.083(-0.093,-0.073)$ & $2.38 \times 10^{-53}$ \\
\hline rs2479409 & $A / G$ & 0.67 & $-0.064(-0.072,-0.056)$ & $2.52 \times 10^{-50}$ \\
\hline rs11591147* & $\mathrm{T} / \mathrm{G}$ & 0.02 & $-0.497(-0.532,-0.462)$ & $8.58 \times 10^{-143}$ \\
\hline rs11206514 & $\mathrm{C} / \mathrm{A}$ & 0.39 & $-0.051(-0.059,-0.043)$ & $9.95 \times 10^{-33}$ \\
\hline rs572512 & $\mathrm{C} / \mathrm{T}$ & 0.65 & $-0.048(-0.057,-0.039)$ & $5.31 \times 10^{-26}$ \\
\hline rs585131 & $\mathrm{C} / \mathrm{T}$ & 0.18 & $-0.064(-0.074,-0.054)$ & $2.70 \times 10^{-35}$ \\
\hline rs12067569* & $\mathrm{G} / \mathrm{A}$ & 0.97 & $-0.089(-0.108,-0.069)$ & $1.97 \times 10^{-17}$ \\
\hline rs10493176 & $\mathrm{G} / \mathrm{T}$ & 0.11 & $-0.078(-0.098,-0.058)$ & $2.54 \times 10^{-14}$ \\
\hline rs11583974* & $G / A$ & 0.97 & $-0.065(-0.088,-0.042)$ & $3.95 \times 10^{-9}$ \\
\hline rs2495477 & $\mathrm{C} / \mathrm{T}$ & NA & $-0.064(-0.075,-0.053)$ & $7.29 \times 10^{-30}$ \\
\hline \multicolumn{5}{|l|}{ LDLR } \\
\hline rs12983316 & $A / G$ & 0.83 & $-0.051(-0.062,-0.041)$ & $7.44 \times 10^{-22}$ \\
\hline rs3786721 & $\mathrm{C} / \mathrm{T}$ & 0.54 & $-0.047(-0.054,-0.039)$ & $2.89 \times 10^{-31}$ \\
\hline rs12052058 & $\mathrm{T} / \mathrm{G}$ & 0.25 & $-0.075(-0.083,-0.067)$ & $9.66 \times 10^{-62}$ \\
\hline rs6511720 & $\mathrm{T} / \mathrm{G}$ & 0.10 & $-0.221(-0.233,-0.209)$ & $1.00 \times 10^{-200}$ \\
\hline rs73015030* & $A / G$ & 0.03 & $-0.152(-0.181,-0.123)$ & $2.62 \times 10^{-22}$ \\
\hline rs1799898 & $\mathrm{T} / \mathrm{C}$ & 0.15 & $-0.033(-0.044,-0.023)$ & $1.96 \times 10^{-9}$ \\
\hline rs688 & $\mathrm{C} / \mathrm{T}$ & 0.55 & $-0.054(-0.061,-0.047)$ & $1.01 \times 10^{-43}$ \\
\hline rs2738464 & $\mathrm{G} / \mathrm{C}$ & 0.13 & $-0.042(-0.054,-0.030)$ & $2.73 \times 10^{-10}$ \\
\hline rs5742911 & $\mathrm{G} / \mathrm{A}$ & 0.27 & $-0.061(-0.072,-0.049)$ & $4.83 \times 10^{-24}$ \\
\hline rs892114 & $\mathrm{G} / \mathrm{A}$ & 0.77 & $-0.035(-0.045,-0.026)$ & $7.63 \times 10^{-13}$ \\
\hline rs7251031 & $\mathrm{T} / \mathrm{G}$ & 0.71 & $-0.046(-0.055,-0.037)$ & $6.24 \times 10^{-23}$ \\
\hline rs379309 & $\mathrm{T} / \mathrm{C}$ & 0.50 & $-0.031(-0.039,-0.024)$ & $1.39 \times 10^{-13}$ \\
\hline \multicolumn{5}{|l|}{ CETP } \\
\hline rs12448528 & $\mathrm{G} / \mathrm{A}$ & 0.77 & $-0.037(-0.047,-0.027)$ & $1.06 \times 10^{-12}$ \\
\hline rs247616 & $\mathrm{T} / \mathrm{C}$ & 0.29 & $-0.055(-0.063,-0.047)$ & $2.57 \times 10^{-37}$ \\
\hline rs1864163 & $\mathrm{G} / \mathrm{A}$ & 0.73 & $-0.044(-0.053,-0.035)$ & $7.97 \times 10^{-21}$ \\
\hline rs9989419 & $\mathrm{G} / \mathrm{A}$ & 0.59 & $-0.028(-0.035,-0.020)$ & $2.49 \times 10^{-12}$ \\
\hline rs12920974 & $\mathrm{G} / \mathrm{T}$ & 0.68 & $-0.032(-0.043,-0.021)$ & $2.96 \times 10^{-8}$ \\
\hline rs9929488 & $\mathrm{G} / \mathrm{C}$ & 0.70 & $-0.037(-0.047,-0.028)$ & $8.15 \times 10^{-13}$ \\
\hline rs118146573 & $\mathrm{G} / \mathrm{A}$ & 0.87 & $-0.053(-0.069,-0.038)$ & $1.02 \times 10^{-10}$ \\
\hline rs289714 & $A / G$ & 0.79 & $-0.036(-0.046,-0.025)$ & $2.85 \times 10^{-10}$ \\
\hline \multicolumn{5}{|l|}{ APOB } \\
\hline rs4665788 & $\mathrm{C} / \mathrm{T}$ & 0.77 & $-0.067(-0.075,-0.058)$ & $1.12 \times 10^{-52}$ \\
\hline rs11685356* & $\mathrm{C} / \mathrm{T}$ & 0.77 & $-0.052(-0.060,-0.043)$ & $1.21 \times 10^{-31}$ \\
\hline rs6754295 & $\mathrm{G} / \mathrm{T}$ & 0.26 & $-0.063(-0.071,-0.055)$ & $1.64 \times 10^{-47}$ \\
\hline rs6725189 & $\mathrm{T} / \mathrm{G}$ & 0.23 & $-0.060(-0.069,-0.052)$ & $5.63 \times 10^{-40}$ \\
\hline
\end{tabular}

(Continued)
TABLE 1 | Continued

\begin{tabular}{|c|c|c|c|c|}
\hline SNP & EA/NA & EAF & Effect $(95 \% \mathrm{Cl})^{\dagger}$ & $P$-value \\
\hline rs533617* & $\mathrm{C} / \mathrm{T}$ & 0.05 & $-0.141(-0.160,-0.121)$ & $9.63 \times 10^{-45}$ \\
\hline rs3791981 & $\mathrm{G} / \mathrm{A}$ & 0.12 & $-0.094(-0.107,-0.081)$ & $2.03 \times 10^{-41}$ \\
\hline rs12691202* & $\mathrm{T} / \mathrm{C}$ & 0.05 & $-0.097(-0.119,-0.074)$ & $8.22 \times 10^{-19}$ \\
\hline rs12720842* & $\mathrm{T} / \mathrm{C}$ & 0.98 & $-0.099(-0.122,-0.077)$ & $1.88 \times 10^{-15}$ \\
\hline rs12720796* & $\mathrm{A} / \mathrm{C}$ & 0.98 & $-0.091(-0.119,-0.063)$ & $1.68 \times 10^{-10}$ \\
\hline rs1367117 & $\mathrm{G} / \mathrm{A}$ & 0.71 & $-0.119(-0.126,-0.111)$ & $9.48 \times 10^{-183}$ \\
\hline rs17398765 & $A / G$ & 0.93 & $-0.092(-0.107,-0.077)$ & $3.54 \times 10^{-32}$ \\
\hline rs7567653* & $A / G$ & 0.04 & $-0.115(-0.136,-0.093)$ & $3.37 \times 10^{-26}$ \\
\hline rs515135 & $\mathrm{T} / \mathrm{C}$ & 0.22 & $-0.139(-0.149,-0.130)$ & $1.09 \times 10^{-178}$ \\
\hline rs6756743* & $\mathrm{C} / \mathrm{T}$ & 0.96 & $-0.055(-0.073,-0.037)$ & $4.97 \times 10^{-9}$ \\
\hline rs113588790* & $\mathrm{C} / \mathrm{T}$ & 0.98 & $-0.090(-0.118,-0.061)$ & $3.94 \times 10^{-9}$ \\
\hline
\end{tabular}

*These SNPS were not available in the RCC GWAS datasets, including three SNPS in PCSK9 (rs11591147, rs12067569, rs 11583974), one SNP in LDLR (rs73015030), and seven SNPs in APOB (rs11685356, rs1291202, rs12720842, rs12720796, rs7567653, rs6756743, rs113588790)

${ }^{\dagger}$ Unit: $38.67 \mathrm{mg} / \mathrm{dL}(1 \mathrm{SD})$.

EAf, Effect Allele Frequency; HMGCR, 3-Hydroxy-3-Methyl-Glutaryl-Coenzyme A Reductase; NPC1L1, Niemann-Pick C1-Like 1; PCSK9, Proprotein Convertase Subtilisin/Kexin type 9; LDLR, Low Density Lipoprotein Receptor; CETP, Cholesteryl Ester Transfer Protein; APOB, Apolipoprotein B.

subtypes. And all controls were healthy participants recruited from large cohort studies. First, for the overall analysis, we used raw genotyping data obtained from $\mathrm{dbGaP}$ (phs000351.v1.p1), which recruited 1,311 cases and 3,424 controls of European ancestry conducted by the National Cancer Institute (NCI). We performed quality control, imputation, and association test as described in (20), and obtained association results of $\sim 6.5$ million SNPs. The data process procedure was presented in Supplementary Figure 1. In addition, we obtained summary genetic association statistics from a recently published sexspecific RCC GWAS (21). For sex-specific analysis, we obtained summary statistics from a publicly available dataset consisting of two IARC-Center National de Genotypage (CNG) scans with 5,219 RCC cases (1,992 women and 3,227 men) and 8,011 controls (3,095 women and 4,916 men) of European ancestry. IARC-2 study, the mean (SD) age of the participants was 60.16 (11.19) years. Quality control, imputation, and sex-specific association analyses protocols were described in a previous study (21). Finally, $\sim 6.4$ million SNPs were retained in the sex-specific GWAS dataset.

\section{Power Calculation and $\boldsymbol{F}$-Statistic}

Statistical power and $F$-statistics were calculated to ensure sufficient statistical power and avoid weak instrument bias. Power calculation was performed using online tools mRnd (http://cnsgenomics.com/shiny/mRnd) (22, 23). The statistical powers to capture an OR of 0.50 per one SD change in the circulating LDL-C levels were shown in Supplementary Table 3. The strength of each instrument was assessed by calculating $F$ statistics; typically, $F$-statistic $>10$ was considered to be no weak 
instrument bias (24).

$$
F=\frac{N-k-1}{k} \times \frac{R^{2}}{1-R^{2}}
$$

$N$ indicated the sample size of the exposure factor, $k$ indicated the number of SNPs in each instrument, and $R^{2}$ represented the variance explained by the instrument. $R^{2}$ was calculated according to the equation proposed by Shim et al. (25).

\section{Statistical Analysis}

Data for SNP associations with LDL-C and with risk of RCC outcomes were harmonized to match coded effect alleles consistently. If the variants were not available for the outcomes, we searched for a proxy $\left(r^{2} \geq 0.8\right)$ for these SNPs. However, if no proxies were founded, the SNPs were omitted. Ambiguous SNPs with palindromic genotypes and minor allele frequencies between 0.4 and 0.5 were excluded from the analysis. We used the multiplicative random effect inverse-variance weighted (IVW) method to generate the overall estimate of causal effect when there are three or more variants in the instruments; while if there are two or fewer variants in the instruments, Wald ratio estimates were used. All the reported ORs of RCC risk were corresponding to one SD of LDL-C levels. In addition, for drugtarget MR analysis, as there was weak $\mathrm{LD}\left(r^{2} \leq 0.2\right)$ among the instruments, we included the correlation matrix of variants that were calculated based on the 1,000 Genomes Phase 3 reference panel for correction. We totally performed 18 tests and applied Benjamini-Hochberg false-discovery rate (FDR) procedure to adjust the raw $p$-values for multiple testing. All statistical tests were two-sided and a significant threshold was set to $P<0.05$. We calculated the $p$-values for the sex disparity in effect estimates (based on log ORs and SEs for RCC). Specifically, we used a wellestablished formula to calculate the $z$ statistics and then obtained the two-tailed $p$-values.

$$
z=\frac{\left(b_{1}-b_{2}\right)}{\sqrt{\left(S E_{b_{1}}^{2}+S E_{b_{2}}^{2}\right)}}
$$

where $b_{1}$ and $b_{2}$ are the MR effect estimates (log ORs and SEs for RCC), and $\mathrm{SE}_{\mathrm{b} 1}$ and $\mathrm{SE}_{\mathrm{b} 2}$ are the standard error of $\mathrm{b}_{1}$ and $\mathrm{b}_{2}$. Statistical analyses were conducted using the TwoSampleMR (v. 0.5.5), MVMR (v. 0.2.0), MendelianRandomization (v. 0.5.0), and MR-PRESSO (v. 1.0) packages in R (v. 3.5.3).

\section{Test of Basic MR Assumptions}

Our study was based on a two-sample MR framework, which obtained SNP-exposure associations and SNP-outcome associations from diverse populations and sources to estimate the causal effects of exposure on the outcome (26). Therefore, three assumptions should be satisfied: (1) a strong link between genetic predictor and the exposure ("relevance"); (2) genetic predictor of the exposure is independent of the confounders influencing the relationship of exposure and outcome ("independence"); (3) genetic predictor affects the outcome only through the exposure of interest ("exclusion restriction"). We conducted three tests to test each assumption mentioned above. First, we applied adaption of $I^{2}$-statistics (referred to as $I_{\mathrm{GX}}^{2}$ ) to test whether there was no measurement error (i.e., NOME assumption) in the SNP-exposure association estimates. $I_{\mathrm{GX}}^{2}$ statistics provided an estimation of the degree of expected relative bias (or dilution) in the MR-Egger causal estimate due to uncertainty in the SNPexposure estimates (27). Simulation extrapolation (SIMEX) was then used to counteract the MR-Egger estimate for this dilution (28). Second, colocalization analysis was applied to test the independence assumption. Colocalization analysis investigated whether SNPs associated with both the exposures and outcomes were shared casual variants. This analysis was carried out with the eCAVIAR package and a threshold of colocalization posterior probability (CLPP) $<0.01$ was set to indicate a significant shared causal variant between drug-target instruments and RCC outcome (29). Third, multivariable MR analysis was applied to test the exclusion-restriction assumption. We included eight established risk factors (e.g., smoking, alcohol consumption; body mass index, BMI; waist-to-hip ratio; height; hypertension; diabetes; and chronic kidney disease) in the univariable MR model to assess the relationship between genetically proxied inhibition of drug targets and these RCC risk factors. We used multivariable MR analysis to adjust for statistically significant risk factors associated with genetically proxied inhibition of drug targets. Consistency of causal relationships between the adjusted and unadjusted model indicated a robust association that unlikely to be biased by other causal pathways (30). Extended descriptions of multivariable MR analyses were shown in Supplementary Methods.

\section{Sensitivity Analysis}

Sensitivity analyses were performed using MR-Egger (31), weighted median (32), weighted mode, and MR-PRESSO (33) methods. Specifically, as there was weak LD $\left(r^{2} \leq 0.2\right)$ among instruments in the drug-target MR analysis, the residual genetic correlation was accounted for when applying these sensitivity analysis methods. MR-Egger intercept test was used to assess heterogeneity between causal effects of individual genetic variants. A pleiotropy test was applied to assess horizontal pleiotropy (34). In addition, we performed leaveone-out analyses to examine whether the removal of one SNP from the instruments iteratively influenced the overall estimates of a causal effect. To help validate the drug-target instrument construction strategies, we also used the same set of SNPs to assess the effect of LDL-C lowering on CAD. In this analysis (i.e., positive control analyses), we expected to see a significant causal effect of LDL-C lowering on CAD risk. Summary GWAS data on CAD risk were obtained from the CARDIoGRAM consortium ( $N=22,233$ cases, 64,762 controls) (35). All participants were European ancestry populations. Moreover, we also replicated the analysis using instrument variables constructed in previous MR studies (36-38). Information of SNPs to construct these genetic scores were listed in Supplementary Tables 13-17.

\section{RESULTS}

\section{MR Estimates}

An overview of the study design was provided in Figure 1. Information of genetic variants in HMGCR, NPC1L1, PCSK9, 
$L D L R, C E T P$, and $A P O B$ used to proxy the effect of drug-target genes were presented in Table $\mathbf{1}$ and Supplementary Table 1. Information of single nucleotide polymorphisms (SNPs), which used to generate instruments of LDL-C, HDL-C, TG, TC, ApoA, and $A p o B$ were listed in Supplementary Table 2. Across the six drug-target instruments examined, $F$-statistics ranged from 64.2 to 369.6 , indicating that weak instrument bias was unlikely to contribute to the analyses. Variance explained by the instruments ranged from 0.13 to $1.51 \%$. For circulating lipid biomarkers analyses, the $F$-statistics of the instruments ranged from 68.1 to 153.6 , and the explained variance ranged from 4.81 to $7.52 \%$ (Supplementary Table 3). The statistical power to capture an OR of 0.50 per one SD change in the circulating LDL-C levels in drug-target $\mathrm{MR}$ analyses was relatively low, ranging from 0.10 to 0.94 .

Results for the genetically predicted lipid-lowering drug targets on RCC and CAD risk adjusting for weak LD among variants were shown in Figure 2 and Supplementary Table 4. All these six drug targets were significantly associated with CAD risk $(P<0.05)$, indicating that the instruments were valid (Supplementary Table 4). We did not observe a significant association between genetically proxied HMGCR inhibition, which was equivalent to one SD reduction in LDL-C, and RCC risk [Odds ratio $[\mathrm{OR}]=1.42,95 \% \mathrm{CI}, 0.29-6.99, P=0.668$ ]; and this association remained non-significant when classified by sex [men: $\mathrm{OR}=1.25$ [95\% CI, 0.56-2.79], $P=0.583$; women: $\mathrm{OR}=0.97$ [95\% CI: 0.36-2.66], $P=0.956$ ]. Similar results were observed in MR analyses for NPC1L1 $(P=0.245), \operatorname{LDLR}(P=$ $0.832)$, and $A P O B(P=0.140)$ inhibitors.

We found that genetically proxied CETP inhibition was significantly associated with a higher risk of RCC $[\mathrm{OR}=18.8$ [95\% CI, 2.45-143.9], $P=4.79 \times 10^{-4}$, FDR-corrected $P=$ 0.006]. Sex-specific analysis indicated that the association was nominally significant in women $[\mathrm{OR}=4.31$ [95\% CI, 1.19-15.6], $P=0.026$, FDR-corrected $P=0.112]$ rather than in men $[\mathrm{OR}=$ 2.16 [95\% CI, 0.77-6.11], $P=0.145$, FDR-corrected $P=0.305]$; however, the difference by sex was not significant $\left(P_{\text {difference }}=\right.$ 0.41 ). In addition, although genetically proxied PCSK9 inhibition was not associated with RCC risk in the overall population [OR = 1.55 [95\% CI, 0.54-4.48], $P=0.421$, FDR-corrected $P=0.520$ ],

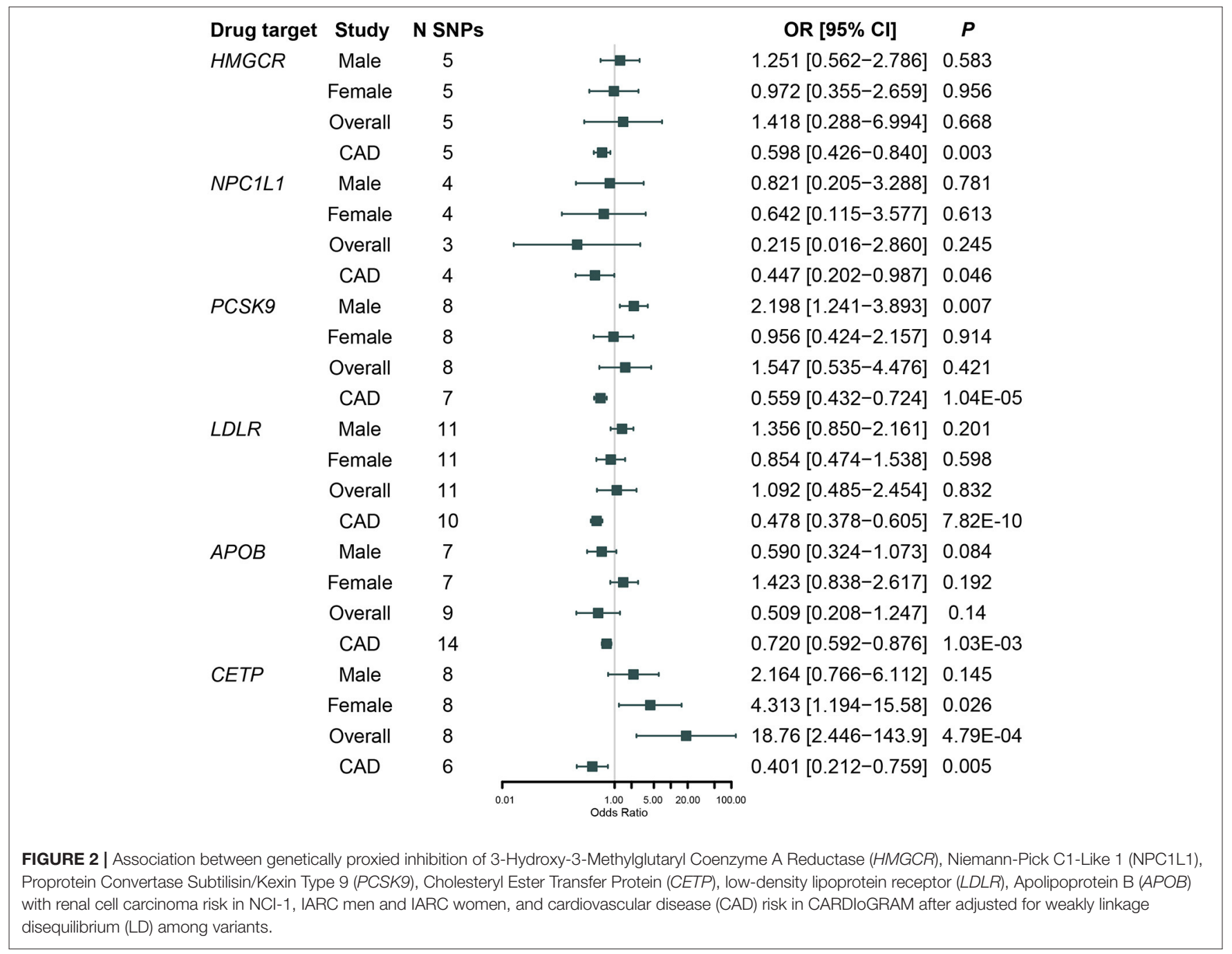


it was significantly associated with a higher risk of RCC in men [OR $=2.20$ [95\% CI, 1.24-3.89], $P=0.007$, FDR-corrected $P=$ 0.045 ] but not in women [OR $=0.96$ [95\% CI, 0.42-2.16], $P=$ 0.914 , FDR-corrected $P=0.701]$; and the difference by sex was moderate $\left(P_{\text {difference }}=0.10\right)$.

Forest plots showed the casual effect estimates of each SNP in PCSK9 and CETP inhibition instruments on RCC in both men and women (Figure 3). Forest plots for other drug targets were shown in Supplementary Figure 2. We found that all the effect estimates of the SNPs in the PCSK9 inhibition instrument on RCC risk in men were consistent except rs11206541. While all effect estimates of the SNPs in the CETP inhibition instrument on RCC risk in women were directionally consistent.

In the secondary analysis, we observed a nominally significant association between circulating TC level and RCC risk in men $[\mathrm{OR}=1.20$ [95\% CI, 1.00-1.44], $P=0.044]$. None of the other circulating traits was significantly associated with overall and sex-specific RCC risk (Supplementary Table 5).

\section{Basic MR Assumption Test Results}

We found that none of the drug-target and lipid trait instruments was departed from the NOME assumption (all $I_{\mathrm{GX}}^{2}>70 \%$, Supplementary Table 6). We performed SIMEXextrapolation MR-Egger tests to counteract the dilution and found consistent results with the standard MR-Egger method (Supplementary Table 7).

We observed no evidence that there were shared causal variants between LDL-C and RCC risk in men at PCSK9 locus (rs11206510, CLPP $=0.004)$ and in women at CETP locus (rs247616, CLPP $=0.004)$ for a-priori defined CLPP threshold of 0.10. Detail results were shown in Supplementary Table 8.

Univariable MR analysis results of genetically proxied inhibition of drug targets and RCC risk factors were shown in Supplementary Table 9. We found that there was evidence for causal associations between PCSK9 inhibitor instrument and lifetime smoking index $[\beta=0.02$ [95\% CI, 0.00, 0.03]; $P=$ 0.028 ]; waist-to-hip ratio ( $\beta=0.05$ [95\% CI, $0.03,0.07$ ]; $P=$ $\left.7.26 \times 10^{-6}\right]$; type 2 diabetes risk [OR $=1.28$ [95\% CI, 1.091.51]; $P=0.002$ ]. In addition, CETP inhibitor instrument was significantly associated with BMI $[\beta=-0.06[95 \% \mathrm{CI},-0.11$, $-0.01] ; P=0.017]$; height $[\beta=-0.06$ [95\% CI, $-0.11,-0.01]$; $P=0.025]$; systolic pressure $[\beta=-1.31$ [95\% CI, -2.19 , $-0.42] ; P=0.004]$; and diastolic pressure $[\beta=-0.59[95 \%$ CI, $-1.10,-0.074] ; P=0.025]$. Results of multivariable MR analysis adjusted for those significant risk factors were shown in Supplementary Table 10. We found that casual associations of genetically proxied PCSK9 inhibition with RCC risk in men remained significant after adjusting for waist-to-hip ratio [OR

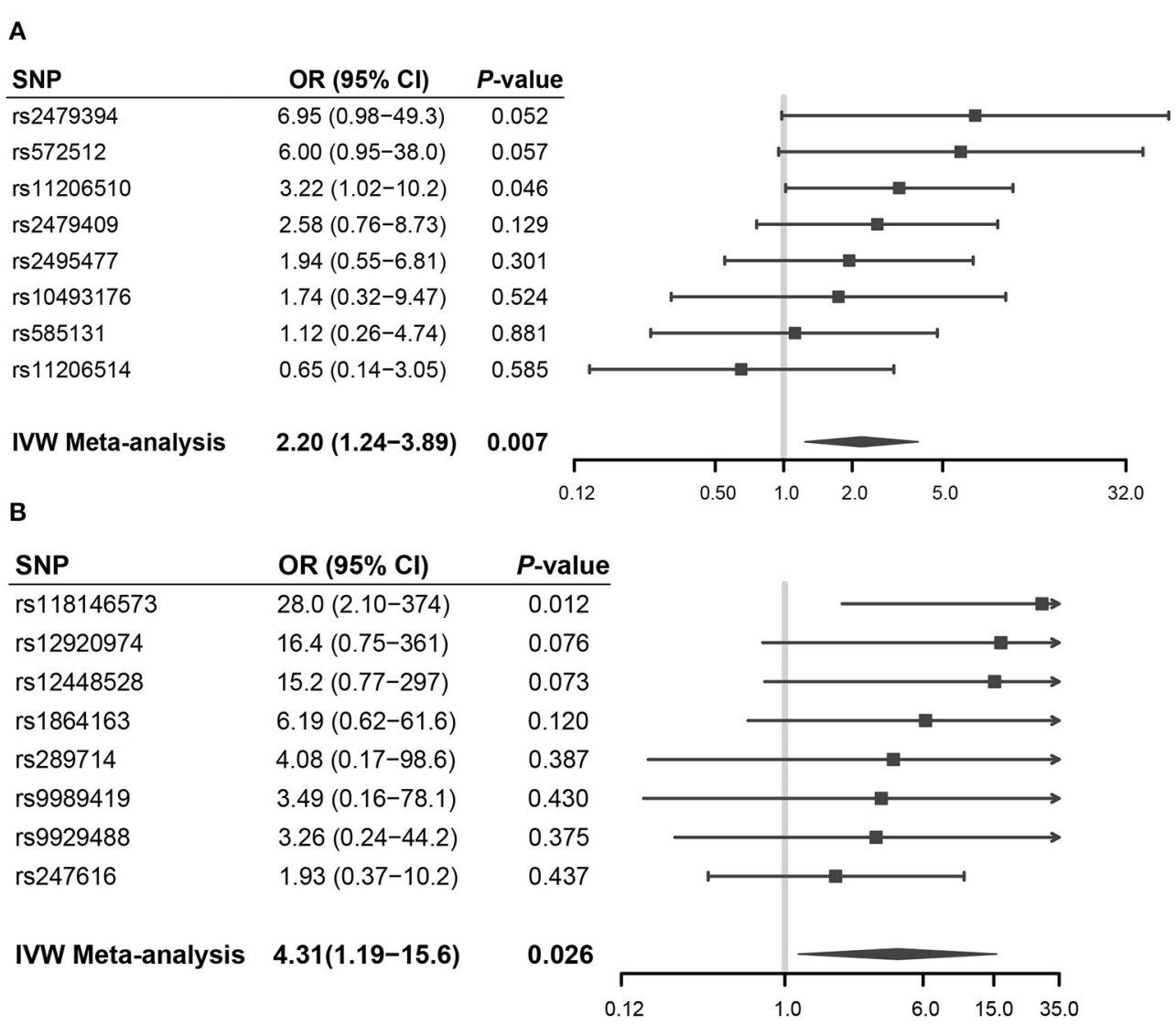

FIGURE 3 | Forest plots showed the casual effect estimates of each SNP in PCSK9 and CETP inhibition instruments on RCC in men and women, respectively. (A) PCSK9 inhibitor and RCC risk in men; (B) CETP inhibitor and RCC risk in women. 
$=1.59$ [95\% CI, 1.02-2.49], $P=0.041$ ], lifetime smoking index [OR $=1.73$ [95\% CI, 1.02-2.93], $P=0.042$ ], and type 2 diabetes $[\mathrm{OR}=2.07$ [95\% CI, 1.33-3.24], $P=0.001]$. However, the causal associations of genetically proxied CETP inhibition with RCC risk in women attenuated substantially after adjusting for BMI $(P$ $=0.409)$, height $(P=0.647)$, systolic pressure $(P=0.985)$, and diastolic pressure $(P=0.765)$.

\section{Sensitivity Analysis}

MR-Egger, weighted median, and weighted mode results were presented in Supplementary Tables 4, 5. For sensitivity analysis of drug-target MR, LD among SNPs was considered. We found that the effect estimates were consistent across these pleiotropyrobust methods.
Results of the leave-one-out analysis were shown in Figure 4 and Supplementary Figure 3. We found that causal effect estimates of genetically proxied inhibition of PCSK9 with RCC risk in men and CETP with RCC risk in women were stable regardless of removal of any SNP in the instruments.

Heterogeneity and pleiotropy test results were presented in Supplementary Tables 11, 12. We observed some evidence for heterogeneity when assessing the causal effect of HDL-C and RCC risk in men (IVW $Q=115.95, P=0.01$; MR-Egger $Q=$ $115.52, P=0.01)$. Thus, we applied the multiplicative randomeffect IVW meta-analysis method and found a consistent result [IVW OR $=1.20$ [95\% CI, 0.97-1.50], $P=0.093$ ]. Pleiotropy tests showed no existence of significant horizontal pleiotropy (all $P>0.05)$.

\section{A}

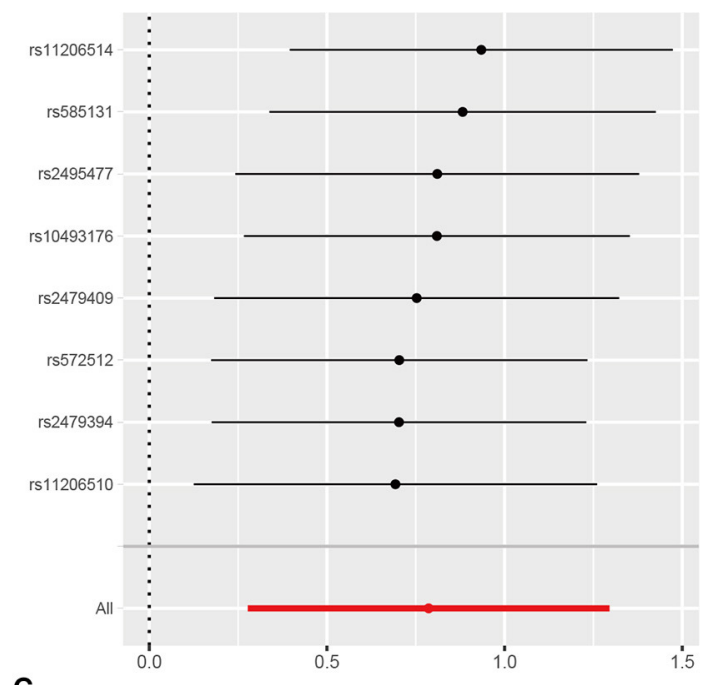

C

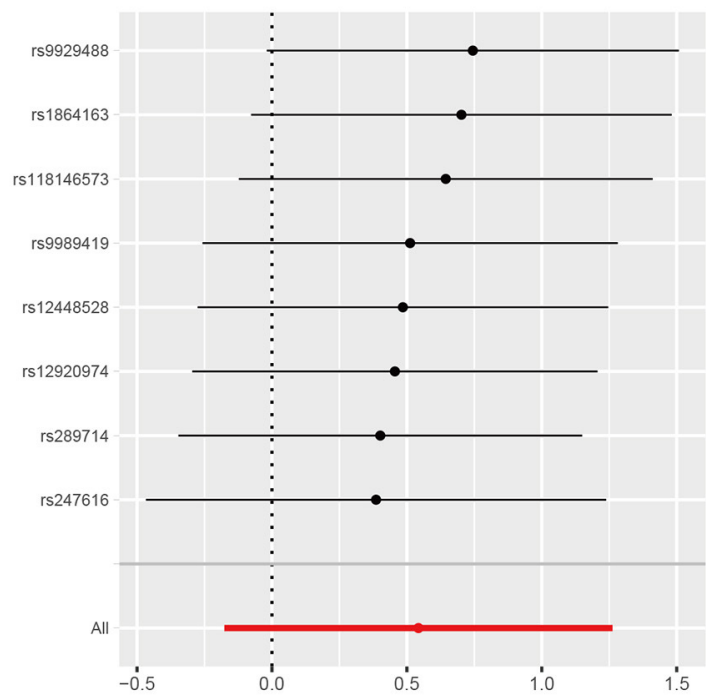

B

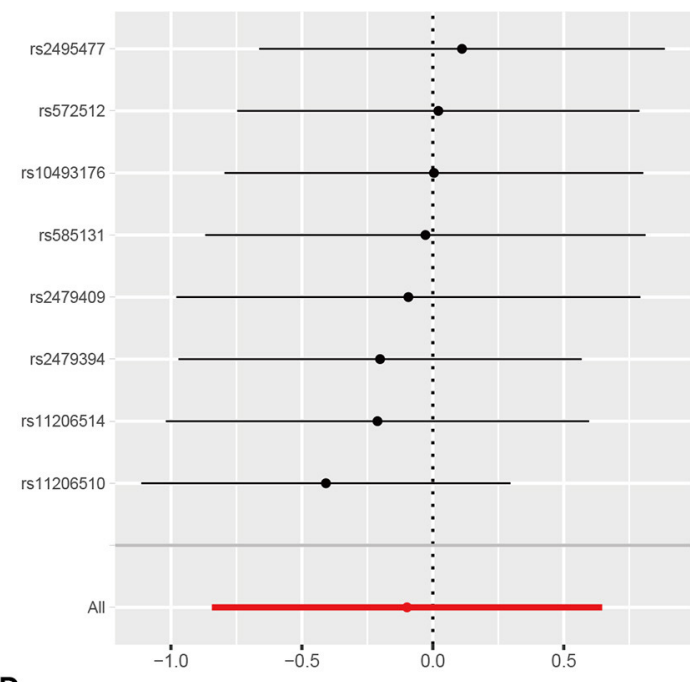

D

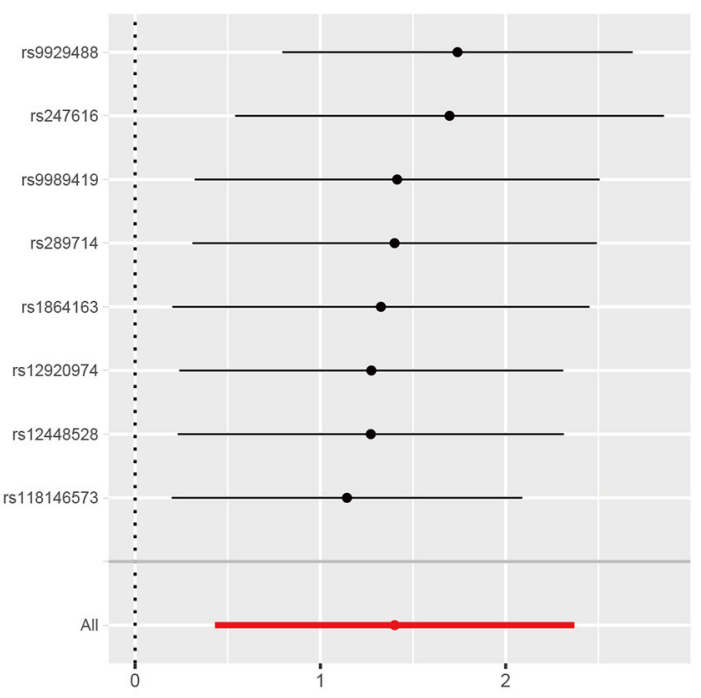

FIGURE 4 | Leave-one-out analysis of genetically proxied PCSK9 and CETP inhibition on RCC risk in men and women. (A) PCSK9 inhibitor and RCC risk in men; (B) PCSK9 inhibitor and RCC risk in women; (C) CETP inhibitor and RCC risk in men; (D) CETP inhibitor and RCC risk in women. 
In comparison analysis using lipid-lowering drug instruments previously reported, we also found that genetically proxied PCSK9 inhibition was causally associated with RCC risk in men $[\mathrm{OR}=1.02$ [95\% CI, 1.00-1.04], $P=0.026]$ (Supplementary Table 18).

\section{DISCUSSIONS}

In this two-sample MR analysis involving 6,530 RCC cases and 11,435 controls of European ancestry, we found that genetically proxied long-term modulation of LDL-C levels by targeting HMGCR genes was not causally associated with reduced risk of RCC. However, genetically proxied PCSK9 inhibition was causally associated with a higher risk of RCC in men, but the difference by sex was not significant. In addition, very limited evidence was shown for an influence of circulating lipid traits, including LDL-C, HDL-C, TC, TG, ApoA, and ApoB, on the risk of RCC, which was consistent with the previous MR study.

\section{Association of HMGCR and RCC Risk}

We observed no evidence of a protective effect of LDL-C lowering and genetically proxied inhibition of HMGCR on RCC risk. This result was directionally consistent with a recent MR study of 1,310 kidney cancer patients from UK Biobank, which found that genetically proxied inhibition of HMGCR was not associated with RCC risk [OR = 1.35 [95\% CI, 0.51-3.57], $P=0.554$ ] (39). Moreover, an umbrella review that reanalyzed the effect of statin on the risk of kidney cancer based on 4,052,120 participants from 11 studies has graded the evidence degree as non-significant (40). In addition, Gebhard et al. have reported that there was no increase in the HMGCR activity in RCC (7). However, it was worth noting that our findings were limited to the influence of LDL-C lowering effect of statins on RCC risk, and did not consider other effects of statins that could also influence the incidence of RCC. For example, it was reported that fluvastatin could potentiate the anticancer activity of vorinostat in renal cancer cells by activating the mechanistic target of rapamycin (mTOR) inhibitor (AMP)-activated protein kinase adenosine monophosphate (AMPK) (41). Therefore, further researches should focus on the potential mechanisms of statins on RCC risk beyond the LDL-C lowering effect.

\section{Association Between PCSK9 and RCC Risk}

It is established that PCSK9 modulates lipid metabolism through degrading LDLR on the surface of hepatocytes (42). However, we found that genetically proxied LDLR inhibition was not causally associated with RCC risk. These results suggested that the mechanisms of PCSK9 inhibitors on RCC risk might be independent of LDL receptor degradation pathways. Previous genetic studies of PCSK9 inhibition suggested that reduced LDLC levels by inhibiting PCSK 9 activity were significantly associated with a higher incidence of diabetes $(43,44)$. Hyperglycemia may contribute to the carcinogenesis of kidney cancer by dysregulation of the rennin-angiotensin system and AMPactivated protein kinase pathways $(45,46)$. In addition, PCSK9 is expressed in the kidney, and involved in nephrogenesis; however, the function of PCSK9 in the kidney remains largely unknown
(47). In vivo study indicated that PCSK9 interacted with epithelial sodium channel $(\mathrm{ENaC})$ subunits and decreased their trafficking to the human embryonic kidney (HEK) 237 cell surface (48). Therefore, inhibiting PCSK9 might promote the trafficking of $\mathrm{ENaC}$, while ENaCs are critically engaged in cancer cell biology, such as proliferation, migration, invasion, and apoptosis (49). Unfortunately, due to the lower prevalence and distinct pathological characteristics of RCC, and the shorter duration of the PCSK9 inhibitor trials, there were few epidemiology studies focused on the association of PCSK9 inhibitors and RCC risk. Further pharmacological researches uncovering the influence of PCSK9 inhibitors on the physiological function of renal cells are warranted. More genetic and observational studies are needed to elucidate the associations.

Interestingly, we found that genetically proxied PCSK9 inhibitor was only significantly associated with RCC risk in men rather than women, and the difference by sex was moderate. Sex disparity of PCSK9 levels has long been discussed. A clinical trial (NCT00848276) explored the relationship among testosterone, estradiol, and circulating PCSK9 levels and suggested that circulating PCSK9 was not related to or affected by testosterone in men, whereas inversely related to estradiol in women (50). Schooling CM et al. have investigated whether statins and PCSK9 inhibitors have pleiotropic effects on ischemic heart disease (IHD) via testosterone in men and women. And they demonstrated genetically proxied effects of statins other than PCSK9 inhibitors in men affected testosterone, which partly mediated effects of IHD (51). It is established that men are at substantially higher lifelong risk of RCC than women ( $\sim 2$-fold). Therefore, it should be more cautious for men with a higher risk of RCC when using PCSK9 inhibitor therapies.

\section{Strength and Limitations}

To our knowledge, our study was the largest drug-target MR analysis so far to explore the causal effect of genetically proxied lipid-modifying agents on overall and sex-specific RCC risk. We comprehensively examined targets of commonly prescribed lipid-lowering drugs, including statins, ezetimibe, evolocumab, anacetrapib, and mipomersen, and a series of sensitivity analyses were applied to test whether the results violated basic assumptions or were biased by horizontal pleiotropy.

However, there were several limitations. The first limitation was the relatively small sample size for RCC risk GWAS studies, which resulted in low statistical power and a wide CI. However, participants in these studies were European ancestry populations recruited from European countries, Australia, and the USA, and they were well-representatives. In the future, larger RCC GWASs and more powerful instruments might enable us to estimate the causal effects more precisely. Second, selection bias might affect MR estimates when samples selected into the study are strongly influenced by the risk factor. However, restricted to the small sample size and insufficient information of the samples, selection bias cannot be ruled out in our study. Future studies with a larger sample size and novel statistical analysis methods (such as inverse probability weighting method) might result in more accurate effect estimates. Third, we could not use sexspecific instrumental variables for circulating lipid traits due to 
limited robust associations between SNP and the traits. However, a recently published sex-specific GWAS across 33 quantitative biomarker traits in UK Biobank demonstrated that sex played a limited role in the genetics of most traits (52). Thus, this bias should have little consequence for the result interpretation. The fourth limitation was that drug-target MR analysis was designed to reflect the effect of life-long modulation of lipidlowering agents to modify LDL-C levels on the disease. It was unable to indicate the effect of short-term administration of lipid-modifying agents. Fifth, all GWAS samples were of mostly European ancestry, making extrapolation of our conclusions to other populations difficult. Sixth, given that the incidence rate of RCC increases steadily with age, with a peak of incidence at $\sim 75$ years, competing risk factors before recruitment might bias the results, which was so-called "winner's curse bias" (53). Seventh, this analysis considered no interaction of the association between genetic variants proxied to the drug targets and RCC risks, such as gene-environment interaction and gene-gene interaction. Eighth, our analysis only revealed the on-target effect of drug use solely and ignored the off-target consequences of related medication. Specifically, we only considered the causal influence of LDL-C lowering effect of lipid-modifying agents on RCC risk; however, the pharmacological mechanisms underlying each medication are more complex. For example, statin use has been reported to lead to extensive lipoprotein and fatty acid changes beyond LDL-C (54). Ninth, some instrument variables were not available in the RCC datasets, which might lead to the missing of the causal effect estimates. However, as these SNPs were in weak LD with each other and leave-one-out analyses were stable regardless of removal of any SNP in the instrument, the missing variants may not change the results substantially.

In conclusion, we found very limited evidence to support the genetically proxied inhibition of HMGCR as a causal protective factor for RCC. However, our results tentatively suggested that genetically proxied inhibition of PCSK9 and CETP were significantly associated with a higher risk of RCC in a sex-specific manner. These findings provided insights into the potential mechanisms of action of the novel lipid-lowering therapies. However, as the pharmacological mechanisms of

\section{REFERENCES}

1. Sung H, Ferlay J, Siegel RL, Laversanne M, Soerjomataram I, Jemal A, et al. Global cancer statistics 2020: GLOBOCAN estimates of incidence and mortality worldwide for 36 cancers in 185 countries. Cancer J Clin. (2021) 71:209-49. doi: 10.3322/caac. 21660

2. Usher-Smith J, Simmons RK, Rossi SH, Stewart GD. Current evidence on screening for renal cancer. Nat Rev Urol. (2020) 17:637-42. doi: 10.1038/s41585-020-0363-3

3. Qu YY, Zhao R, Zhang HL, Zhou Q, Xu FJ, Zhang X, et al. Inactivation of the AMPK-GATA3-ECHS1 pathway induces fatty acid synthesis that promotes clear cell renal cell carcinoma growth. Cancer Res. (2020) 80:31933. doi: 10.1158/0008-5472.CAN-19-1023

4. Van Hemelrijck M, Garmo H, Hammar N, Jungner I, Walldius G, Lambe $\mathrm{M}$, et al. The interplay between lipid profiles, glucose, BMI and risk of kidney cancer in the Swedish AMORIS study. Int J Cancer. (2012) 130:211828. doi: $10.1002 /$ ijc. 26212 these medications were complex, we could not conclude that short-term administration of PCSK9 and CETP inhibitor therapies would increase RCC risk.

\section{DATA AVAILABILITY STATEMENT}

Summary statistics for circulating lipid traits produced by GLGC are available at: http://csg.sph.umich.edu/willer/public/ lipids2013/. All meta-analysis results of circulating metabolic traits quantified by NMR are available through URL: http://www. computationalmedicine.fi/data/NMR_GWAS/. Genotype data of NCI-1 scan are accessible on dbGaP: phs000351.v1.p1. Genomewide sex-specific summary statistics of IARC scan are publicly available through the NHGRI-EBI GWAS Catalog: https://www. ebi.ac.uk/gwas/downloads/summary-statistics.

\section{AUTHOR CONTRIBUTIONS}

All authors listed have made a substantial, direct and intellectual contribution to the work, and approved it for publication.

\section{FUNDING}

This work was supported by the Chinese National Key Research and Development Project (Grant No. 2018YFC1315600) and Tianjin Municipal Education Commission (2016YD21).

\section{ACKNOWLEDGMENTS}

The authors would like to thank GLGC, NCI, and IARC for providing GWAS summary datasets. The authors would also like to acknowledge the $\mathrm{dbGaP}$ repository for providing genotyping datasets. The accession number is phs000351.v1.p1.

\section{SUPPLEMENTARY MATERIAL}

The Supplementary Material for this article can be found online at: https://www.frontiersin.org/articles/10.3389/fnut.2021. 755834/full\#supplementary-material DC, et al. The influence of obesity-related factors in the etiology of renal cell carcinoma-A mendelian randomization study. PLoS Med. (2019) 16:e1002724. doi: 10.1371/journal.pmed.1002724

6. Demierre MF, Higgins PD, Gruber SB, Hawk E, Lippman SM. Statins and cancer prevention. Nat Rev Cancer. (2005) 5:930-42. doi: 10.1038/nrc1751

7. Gebhard RL, Clayman RV, Prigge WF, Figenshau R, Staley NA, Reesey C, et al. Abnormal cholesterol metabolism in renal clear cell carcinoma. J Lipid Res. (1987) 28:1177-84. doi: 10.1016/S0022-2275(20)38606-5

8. Chou YC, Lin CH, Wong CS, Chou WY, Chang JY, Sun CA. Statin use and the risk of renal cell carcinoma: national cohort study. J Investig Med. (2020) 68:776-81. doi: 10.1136/jim-2019-001209

9. Liu W, Choueiri TK, Cho E. Statin use and the risk of renal cell carcinoma in 2 prospective US cohorts. Cancer. (2012) 118:797-803. doi: 10.1002/cncr.26338

10. Pottegård A, Clark P, Friis S, Hallas J, Lund L. Long-term use of statins and risk of renal cell carcinoma: a population-based case-control study. Eur Urol. (2016) 69:877-82. doi: 10.1016/j.eururo.2015.10.020 
11. Kang M, Ku JH, Kwak C, Kim HH, Jeong CW. Effects of Aspirin, nonsteroidal anti-inflammatory drugs, statin, and COX2 inhibitor on the developments of urological malignancies: a population-based study with 10-year follow-up data in Korea. Cancer Res Treat. (2018) 50:984-91. doi: 10.4143/crt.2017.248

12. Bowman L, Hopewell JC, Chen F, Wallendszus K, Stevens W, Collins R, et al. Effects of anacetrapib in patients with atherosclerotic vascular disease. $\mathrm{N} \mathrm{Engl}$ J Med. (2017) 377:1217-27. doi: 10.1056/NEJMoa1706444

13. Davey Smith G, Hemani G. Mendelian randomization: genetic anchors for causal inference in epidemiological studies. Hum Mol Genet. (2014) 23:R8998. doi: 10.1093/hmg/ddu328

14. Gordillo-Marañón M, Zwierzyna M, Charoen P, Drenos F, Chopade $\mathrm{S}$, Shah $\mathrm{T}$, et al. Validation of lipid-related therapeutic targets for coronary heart disease prevention using human genetics. bioRxiv [preprint]. (2020). doi: 10.1101/2020.11.11.377747

15. Schmidt AF, Finan C, Gordillo-Marañón M, Asselbergs FW, Freitag DF, Patel RS, et al. Genetic drug target validation using Mendelian randomisation. Nat Commun. (2020) 11:3255. doi: 10.1038/s41467-020-16969-0

16. Yarmolinsky J, Bull CJ, Vincent EE, Robinson J, Walther A, Smith GD, et al. Association between genetically proxied inhibition of HMGCoA reductase and epithelial ovarian cancer. JAMA. (2020) 323:64655. doi: 10.1001/jama.2020.0150

17. Luo S, Schooling CM, Wong ICK, Au Yeung SL. Evaluating the impact of AMPK activation, a target of metformin, on risk of cardiovascular diseases and cancer in the UK Biobank: a Mendelian randomisation study. Diabetologia. (2020) 63:2349-58. doi: 10.1007/s00125-020-05243-Z

18. Willer CJ, Schmidt EM, Sengupta S, Peloso GM, Gustafsson S, Kanoni S, et al. Discovery and refinement of loci associated with lipid levels. Nat Genet. (2013) 45:1274-83. doi: 10.1038/ng.2797

19. Kettunen J, Demirkan A, Würtz P, Draisma HH, Haller T, Rawal $\mathrm{R}$, et al. Genome-wide study for circulating metabolites identifies 62 loci and reveals novel systemic effects of LPA. Nat Commun. (2016) 7:11122. doi: $10.1038 /$ ncomms 11122

20. Purdue MP, Johansson M, Zelenika D, Toro JR, Scelo G, Moore LE, et al. Genome-wide association study of renal cell carcinoma identifies two susceptibility loci on 2p21 and 11q13.3. Nat Genet. (2011) 43:605. doi: 10.1038/ng.723

21. Laskar RS, Muller DC, Li P, Machiela MJ, Ye Y, Gaborieau V, et al. Sex specific associations in genome wide association analysis of renal cell carcinoma. Eur J Hum Genet. (2019) 27:1589-98. doi: 10.1038/s41431-019-0455-9

22. Freeman G, Cowling BJ, Schooling CM. Power and sample size calculations for Mendelian randomization studies using one genetic instrument. Int J Epidemiol. (2013) 42:1157-63. doi: 10.1093/ije/dyt110

23. Brion MJ, Shakhbazov K, Visscher PM. Calculating statistical power in Mendelian randomization studies. Int J Epidemiol. (2013) 42:1497501. doi: 10.1093/ije/dyt179

24. Haycock PC, Burgess S, Wade KH, Bowden J, Relton C, Davey Smith G. Best (but oft-forgotten) practices: the design, analysis, and interpretation of Mendelian randomization studies. Am J Clin Nutr. (2016) 103:96578. doi: 10.3945/ajcn.115.118216

25. Shim H, Chasman DI, Smith JD, Mora S, Ridker PM, Nickerson DA, et al. A multivariate genome-wide association analysis of $10 \mathrm{LDL}$ subfractions, and their response to statin treatment, in 1868 Caucasians. PLoS ONE. (2015) 10:e0120758. doi: 10.1371/journal.pone.0120758

26. Lawlor DA. Commentary: two-sample Mendelian randomization: opportunities and challenges. Int J Epidemiol. (2016) 45:90815. doi: 10.1093/ije/dyw127

27. Higgins JP, Thompson SG, Deeks JJ, Altman DG. Measuring inconsistency in meta-analyses. BMJ. (2003) 327:557-60. doi: 10.1136/bmj.327.7414.557

28. Bowden J, Del Greco MF, Minelli C, Davey Smith G, Sheehan NA, Thompson JR. Assessing the suitability of summary data for two-sample Mendelian randomization analyses using MR-Egger regression: the role of the I2 statistic. Int J Epidemiol. (2016) 45:1961-74. doi: 10.1093/ije/dyw220

29. Hormozdiari F, van de Bunt M, Segrè AV, Li X, Joo JWJ, Bilow M, et al. Colocalization of GWAS and eQTL signals detects target genes. Am J Hum Genet. (2016) 99:1245-60. doi: 10.1016/j.ajhg.2016.10.003

30. Sanderson E, Davey Smith G, Windmeijer F, Bowden J. An examination of multivariable Mendelian randomization in the single-sample and two-sample summary data settings. Int J Epidemiol. (2019) 48:71327. doi: $10.1093 / \mathrm{ije} /$ dyy262

31. Bowden J, Davey Smith G, Burgess S. Mendelian randomization with invalid instruments: effect estimation and bias detection through Egger regression. Int J Epidemiol. (2015) 44:512-25. doi: 10.1093/ije/dyv080

32. Bowden J, Davey Smith G, Haycock PC, Burgess S. Consistent estimation in mendelian randomization with some invalid instruments using a weighted median estimator. Genet Epidemiol. (2016) 40:304-14. doi: 10.1002/gepi.21965

33. Verbanck M, Chen CY, Neale B, Do R. Detection of widespread horizontal pleiotropy in causal relationships inferred from Mendelian randomization between complex traits and diseases. Nat Genet. (2018) 50:693-8. doi: 10.1038/s41588-018-0099-7

34. Bowden J, Del Greco MF, Minelli C, Davey Smith G, Sheehan N, Thompson J. A framework for the investigation of pleiotropy in twosample summary data Mendelian randomization. Stat Med. (2017) 36:1783802. doi: 10.1002/sim.7221

35. Schunkert H, König IR, Kathiresan S, Reilly MP, Assimes TL, Holm H, et al. Large-scale association analysis identifies 13 new susceptibility loci for coronary artery disease. Nat Genet. (2011) 43:333-8. doi: 10.1038/ ng.784

36. Ference BA, Majeed F, Penumetcha R, Flack JM, Brook RD. Effect of naturally random allocation to lower low-density lipoprotein cholesterol on the risk of coronary heart disease mediated by polymorphisms in NPC1L1, HMGCR, or both: a $2 \times 2$ factorial Mendelian randomization study. J Am Coll Cardiol. (2015) 65:1552-61. doi: 10.1016/j.jacc.2015. 02.020

37. Ference BA, Robinson JG, Brook RD, Catapano AL, Chapman MJ, Neff DR, et al. Variation in PCSK9 and HMGCR and risk of cardiovascular disease and diabetes. N Engl J Med. (2016) 375:2144-53. doi: 10.1056/NEJMoa1604304

38. Ference BA, Kastelein JJP, Ginsberg HN, Chapman MJ, Nicholls SJ, Ray $\mathrm{KK}$, et al. Association of genetic variants related to CETP inhibitors and statins with lipoprotein levels and cardiovascular risk. JAMA. (2017) 318:94756. doi: 10.1001/jama.2017.11467

39. Carter P, Vithayathil M, Kar S, Potluri R, Mason AM, Larsson SC, et al. Predicting the effect of statins on cancer risk using genetic variants from a Mendelian randomization study in the UK Biobank. eLife. (2020) 9:e57191. doi: 10.7554/eLife.57191.sa2

40. Jeong GH, Lee KH, Kim JY, Eisenhut M, Kronbichler A, van der Vliet HJ, et al. Effect of statin on cancer incidence: an umbrella systematic review and meta-analysis. J Clin Med. (2019) 8:819. doi: 10.3390/jcm8060819

41. Okubo K, Isono M, Miyai K, Asano T, Sato A. Fluvastatin potentiates anticancer activity of vorinostat in renal cancer cells. Cancer Sci. (2020) 111:112-26. doi: 10.1111/cas.14225

42. Maxwell KN, Breslow JL. Adenoviral-mediated expression of Pcsk9 in mice results in a low-density lipoprotein receptor knockout phenotype. Proc Natl Acad Sci USA. (2004) 101:7100-5. doi: 10.1073/pnas.0402133101

43. Schmidt AF, Swerdlow DI, Holmes MV, Patel RS, Fairhurst-Hunter Z, Lyall DM, et al. PCSK9 genetic variants and risk of type 2 diabetes: a mendelian randomisation study. Lancet Diabetes Endocrinol. (2017) 5:97105. doi: 10.1016/S2213-8587(16)30396-5

44. Lotta LA, Sharp SJ, Burgess S, Perry JRB, Stewart ID, Willems SM, et al. Association between low-density lipoprotein cholesterol-lowering genetic variants and risk of type 2 diabetes: a meta-analysis. JAMA. (2016) 316:138391. doi: 10.1001/jama.2016.14568

45. Larsson SC, Wolk A. Diabetes mellitus and incidence of kidney cancer: a meta-analysis of cohort studies. Diabetologia. (2011) 54:1013-8. doi: 10.1007/s00125-011-2051-6

46. Yang X, So WY, Ma RC, Kong AP, Xu G, Chan JC. Diabetes and cancer: the mechanistic implications of epidemiological analyses from the Hong Kong Diabetes Registry. Diabetes Metab Res Rev. (2012) 28:37987. doi: $10.1002 / \mathrm{dmrr} .2287$

47. Stoekenbroek RM, Lambert G, Cariou B, Hovingh GK. Inhibiting PCSK9 - biology beyond LDL control. Nat Rev Endocrinol. (2018) 15:5262. doi: 10.1038/s41574-018-0110-5

48. Sharotri V, Collier DM, Olson DR, Zhou R, Snyder PM. Regulation of epithelial sodium channel trafficking by proprotein 
convertase subtilisin/kexin type 9 (PCSK9). J Biol Chem. (2012) 287:19266-74. doi: 10.1074/jbc.M112.363382

49. Liu C, Zhu LL, Xu SG, Ji HL, Li XM. ENaC/DEG in tumor development and progression. J Cancer. (2016) 7:1888-91. doi: 10.7150/jca.15693

50. Ooi TC, Raymond A, Cousins M, Favreau C, Taljaard M, Gavin C, et al. Relationship between testosterone, estradiol and circulating PCSK9: crosssectional and interventional studies in humans. Clin Chim Acta. (2015) 446:97-104. doi: 10.1016/j.cca.2015.03.036

51. Schooling CM, Zhao JV, Au Yeung SL, Leung GM. Investigating pleiotropic effects of statins on ischemic heart disease in the UK Biobank using Mendelian randomisation. eLife. (2020) 9:e58567. doi: 10.7554/eLife.58 567.sa2

52. Flynn E, Tanigawa Y, Rodriguez F, Altman RB, Sinnott-Armstrong N, Rivas MA. Sex-specific genetic effects across biomarkers. Eur J Hum Genet. (2021) 29:154-63. doi: 10.1038/s41431-020-00712-w

53. Schooling CM, Lopez PM, Yang Z, Zhao JV, Au Yeung SL, Huang JV. Use of multivariable mendelian randomization to address biases due to competing risk before recruitment. Front Genet. (2020) 11:610852. doi: 10.3389/fgene.2020.610852

54. Würtz P, Wang Q, Soininen P, Kangas AJ, Fatemifar G, Tynkkynen $\mathrm{T}$, et al. Metabolomic Profiling of Statin Use and Genetic

$$
\begin{aligned}
& \text { Inhibition of HMG-CoA Reductase. J Am Coll Cardiol. } \\
& \text { 67:1200-10. doi: } 10.1016 / \text { j.jacc.2015.12.060 }
\end{aligned}
$$

Conflict of Interest: The authors declare that the research was conducted in the absence of any commercial or financial relationships that could be construed as a potential conflict of interest.

Publisher's Note: All claims expressed in this article are solely those of the authors and do not necessarily represent those of their affiliated organizations, or those of the publisher, the editors and the reviewers. Any product that may be evaluated in this article, or claim that may be made by its manufacturer, is not guaranteed or endorsed by the publisher.

Copyright (C) 2021 Liu, Sheng, Lyu, Dai and Chen. This is an open-access article distributed under the terms of the Creative Commons Attribution License (CC BY). The use, distribution or reproduction in other forums is permitted, provided the original author(s) and the copyright owner(s) are credited and that the original publication in this journal is cited, in accordance with accepted academic practice. No use, distribution or reproduction is permitted which does not comply with these terms. 